$$
\text { "tmcs-klukovits" — 2013/10/21 — 15:33 — page 255 — \#1 }
$$

\title{
On the Past of a famous Theorem: the predecessors of a Theorem of Pythagoras
}

\author{
LAJOS KLUKOVITS
}

\section{Dedicated to Béla Csákány on his 80th birthday}

\begin{abstract}
The well-known Theorem of Pythagoras asserts a relation among the sides of any right-angled triangle. It can be found any secondary school textbook. An interesting question whether this result due to the Pythagoreans from the VIth century BC, or it was known in earlier civilizations. The first answer is a vague yes. According to the legends the Egyptian rope-stretchers used a triangle with sides 3,4,5 units to create right angle. But are there real evidences that this result was known earlier? We will argue that in almost all river-valley civilizations it was known and used.
\end{abstract}

Key words and phrases: ancient mathematics, Theorem of Pythagoras.

ZDM Subject Classification: A30, D20.

1. The Theorem in present day textbooks and in Euclid's book

Consider two formulations of the theorem from the XXth century. In most of the present day textbooks we can read similar drafting.

Theorem A. The sum of the squares of the lenghts of the legs of a right triangle is equal to the square of the hypotenuse [3].

Supported by the European Union and co-funded by the European Social Fund under the project "Telemedicine-focused research activities on the field of Mathematics, Informatics and Medical sciences" of project number "TÁMOP-4.2.2.A-11/1/KONV-2012-0073".

Copyright (C) 2013 by University of Debrecen 


$$
\text { "tmcs-klukovits" — 2013/10/21 — 15:33 — page } 256 \text { — \#2 }
$$

THEOREM B. In a right-angled triangle the square on the hypotenuse is equal to the sum of the squares on the other two sides [2].

Theorem C (Euclid, Elements, Proposition I.47.). In right-angled triangles the square on the side subtending the right angle is equal to the squares on the sides containing the right angle [4].

\section{REMARKS.}

(1) Theorem A has some number theoretic flavour.

(2) The most frequently used textbook's proof of Theorems A or B is based on two different partitions of a square that of the side is the sum of the perpendicular sides of the triangle.

(3) The trouble with this type of proof is that it is very hard to find a connection between the statement and the essence of the argument.

The first deductive proof is probably due to the Pythagoreans. We have no written source on it. Euclid's proof [4] is lucidly geometric and based on the following two propositions of the Elements.

Proposition I.14. If with any straight line, and at a point on it, two straight lines not lying on the same side make adjacent angles equal to two right angles, the two straight lines will be in a straight line with the another.

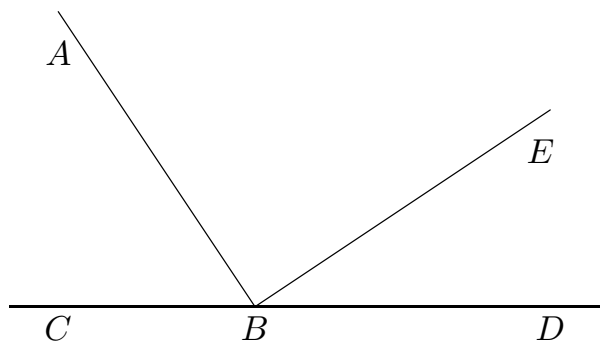

The straight-lines $B C$ and $B D$, not lying on the same side of the line $A B$, make adjacent angles $A B C$ and $A B D$ (whose sum is) equal two right-angles with some straight-line $A B$, at the point $B$. The assertion is that $B D$ is straight-on with respect to $C B$.

Proposition I.41. If a parallelogram have the same base with a triangle and be in the same parallels, the parallelogram is double of the triangle. 


$$
\text { "tmcs-klukovits" — 2013/10/21 — 15:33 — page } 257 \text { — \#3 }
$$

Now we briefly describe the proof of the Pythagorean theorem as was done by Euclid (see also the figure below).

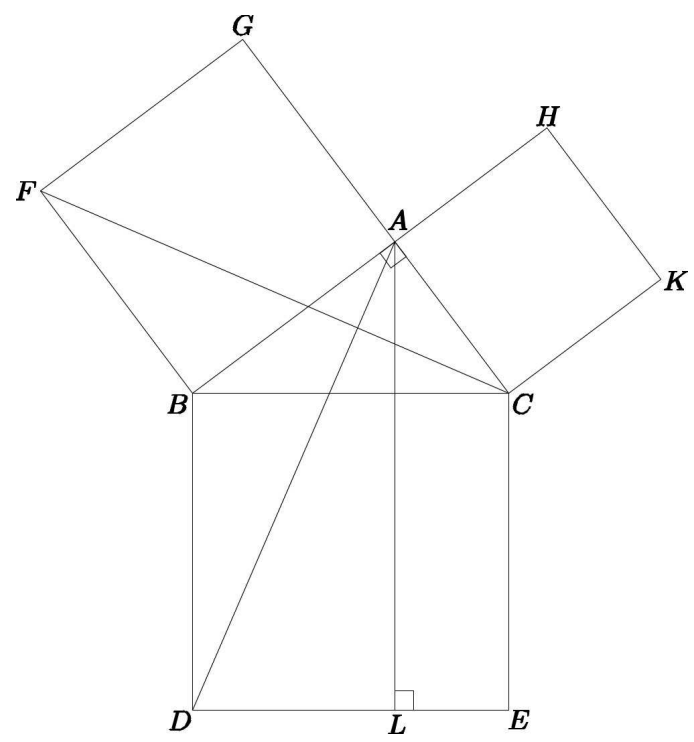

The points $G, A, C$ and $H, A, B$ are on the same lines (Prop. I.14.). The triangles $F B C$ and $A B D$ are congruent. The triangle $F B C$ is a half of the parallelogram $A G F B$ and the same is valid in case of the triangle $A B D$ and the parallelogram determined by the points $B, D, L$ (Prop. I.41.). According to an axiom from Book I the two parallelograms are equal.

Similarly, the square on the side $A C$ is equal to the paralelogram determined by the points $C, E, L$.

While the Egyptians - according to some sources - in the period of the Middle Kingdom knew that a triangle with sides 3,4,5 units is right-angled we can put the following question: Was the result on right-angled triangles known in earlier civizations?

If the answer is yes, what was its form: pure geometric, or had some number theoretic flavour, e.g., the notion of the Pythagorean triple was known.

Definition. A triple of natural numbers (i.e., positive integers) $(a, b, c) \in \mathbb{N}^{3}$ is a Pythagorean triple if $a^{2}+b^{2}=c^{2}$, or in other words, if it is a positive integer solution of the equation

$$
x^{2}+y^{2}=z^{2} .
$$




$$
\text { "tmcs-klukovits" — 2013/10/21 — 15:33 — page 258 — \#4 }
$$

A Pythagorean triple $(a, b, c) \in \mathbb{N}^{3}$ is called primitive if $\operatorname{gcd}(a, b, c)=1$. ( $\mathbb{N}$ denotes the set of natural numbers, i.e., the set of positive integers.)

There are two ancient Greek methods to construct such triples, i.e., to find positive integer solutions of (1).

Rewrite (1) in the form of

$$
(z-y)(z+y)=x^{2}
$$

According to Proclus' commentary to the first book of Euclid's Element [9], a method to solve equation (2) in a special case is ascribed to Pythagoras.

Method 1 (Pythagoras). Consider an odd number $a$ and put

$$
x=a, \quad y=\frac{1}{2}\left(a^{2}-1\right), \quad z=\frac{1}{2}\left(a^{2}+1\right) .
$$

Clearly, in this case $z-y=1$ and $z+y=a^{2}$. The reader can easily prove that all such Pythagorean triples can obtain by Method 1 .

An another special case is when $z-y=2$ in (2). A method ascribed to Plato [10] is the following:

Method 2 (Plato [10]). Consider an even number, say $2 c$. Then

$$
x=2 c, \quad y=c^{2}-1, \quad z=c^{2}+1 .
$$

Now $z-y=2, z+y=2 c^{2}$, and all triples with $z-y=2$ can be obtained by this method.

According to certain sources, the Greeks was familiar two more general methods to get Pythagorean triples.

Method 3 (Archytas of Tarrentum Vth century BC [10]). If $x=s t$ is odd, then from (2)

$$
\begin{array}{ll}
x+z=s^{2}, & z=\frac{1}{2}\left(s^{2}+t^{2}\right), \\
z-y=t^{2}, & y=\frac{1}{2}\left(s^{2}-t^{2}\right) .
\end{array}
$$




$$
\text { "tmcs-klukovits" — 2013/10/21 — 15:33 — page 259 — \#5 }
$$

Method 4 (Diophantus of Alexandria about $250 \mathrm{AD}$ [10]). If $x=2 p q$ is even, then from (2)

$$
\begin{array}{ll}
x+z=2 p^{2}, & z=p^{2}+q^{2}, \\
z-y=2 q^{2}, & y=p^{2}-q^{2} .
\end{array}
$$

\section{Digging deep, step 1: India at about 800 BC}

The Sulvasutras are ancient Hindu manuals. Most of them were composed between 500 and 300 BC, but they contained earlier knowledge mostly. One of the main topic of these sacred collections were altar constructions of different shapes. A Sulvasutra ascribed to Baudhayana contained the following statement[8]:

The diagonal of an oblong produces by itself both the areas which the two sides of the oblong produced separately (i.e., the square of the diagonal is equal to the sum of the squares of the two sides).

This is theorem-like statement, but there was no proof, only a short explanation: This is seen in those oblongs the sides of which are 3 and 4, 12 and 5, 15 and 8, 7 and 24, 12 and 3515 and 36 .

Other sacred collections Sathapata Brahmana and Taittiriya Samhita contain a special altar, called Mahaveni, constructions the shape of which are isosceles trapezoid having bases 24 and 30 and width 36 (units). In the trapezoid one can easily recognize a right-angled triangle with sides 15 , 36, 39, i.e., the Pythagorean triple $(15,36,39)$ appeared here.

If we examine the Mahaveni altar, we can discover other right-angled triangles and Pythagorean triples as well. They are the same as mentioned in Baudhayana's Sulvasutra, see the figure below [8]:
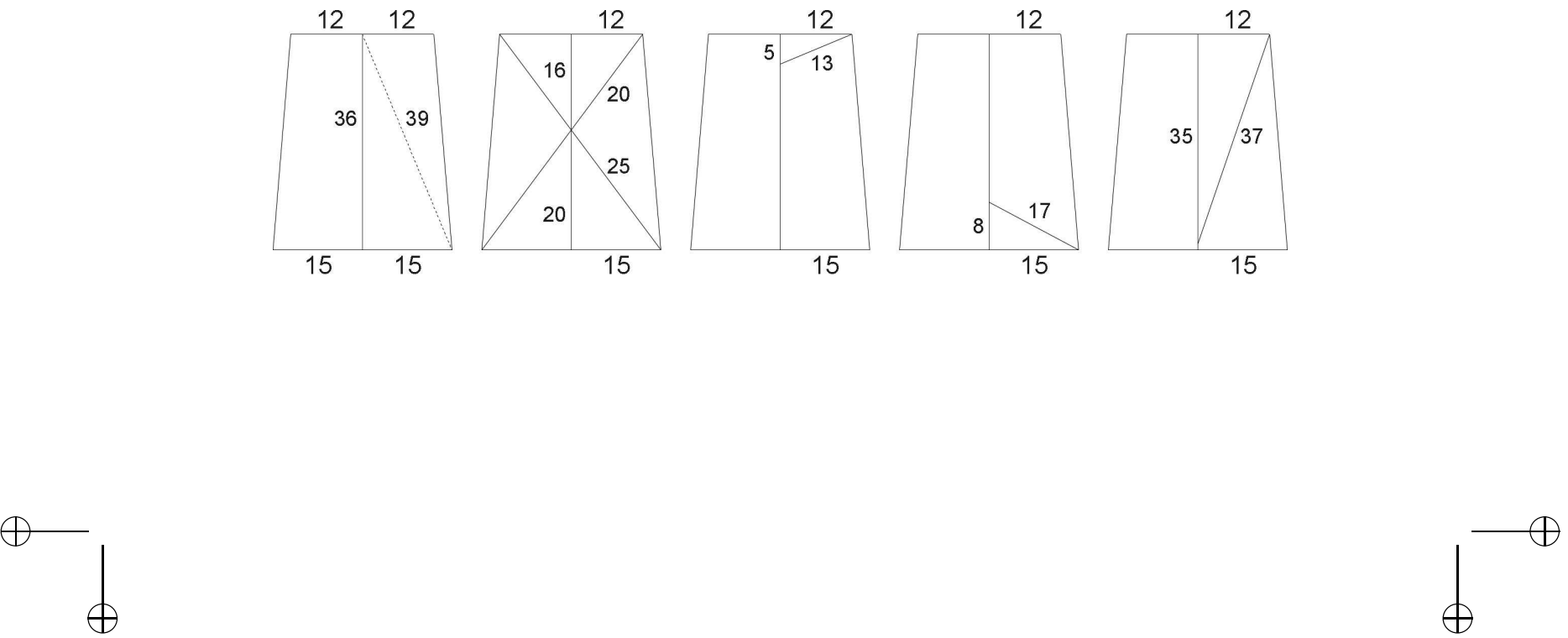


$$
\text { "tmcs-klukovits" — 2013/10/21 — 15:33 — page } 260 \text { — \#6 }
$$

These triples can be classified, using the notations in (2), as

\begin{tabular}{l|c|l}
$z-y=1$ & $z-y=2$ & $z-y=3$ \\
\hline $3,4,5$ & $8,15,17$ & $15,36,39$ \\
$5,12,13$ & $12,35,37$ & \\
$7,24,25$ & &
\end{tabular}

We might suppose that Baudhayana or some of his predecessors obtained this triples empirically simply measuring right-angled triangles. This could be carried out in some cases, but practically impossible to obtain the triple $(12,35,37)$, which might need an enormous number of trials.

But it is easy to see that the triples in the first column can be calculated using the Greek Method 1, while the two triples in the second one via Method 2. The triple $(15,36,39)$ is just 3 times $(5,12,13)$. From this the notion of similar triangles might be appear, or the knowledge that if $(a, b, c)$ is a Pythagorean triple then for any positive integer $d(d a, d b, d c)$ is a Pythagorean triple again.

There is a second possibility to calculate these triples in case of $z-y=1$ or $z-y=2$ using equation (2), which has now the form of

$$
z+y=x^{2}, \quad z+y=\frac{1}{2} x^{2} .
$$

By the method of trial and error the scribe had to find two numbers the sum of which is a square number, e.g., $4+5=9$ or in the second case two such numbers the whose sum is a half of a square number, e.g., $15+17=\frac{1}{2} \cdot 64$.

\section{Digging deep, step 2: China at about 1000 BC}

In the early Han period (from $206 \mathrm{BC}$ to $8 \mathrm{AD}$ ) was a collection of 246 mathematical problem composed, entitled Nine Chapters on the Mathematical Art [1]. This collection is a summary of the Chinese mathematics of the first millennium BC. Chapter IX deals with right-angled triangles.

In Problems 1-3 one side of such triangles is asked knowing the others. The calculations went on to the former equation (1). In each one the triplet $(3,4,5)$ appeared.

Consider two other problems in detail.

Problem IX.4. One have a rounded pole with diameter 2 ch'in 5 ts'un, and want to make a quadrangle beam from it with a height of 7 ts'un. What can be its width? 


$$
\text { "tmcs-klukovits" — 2013/10/21 — 15:33 — page } 261 \text { — \#7 }
$$

Answer. 2ch'in $4 \frac{5}{10}$ ts'un. (1 ch'in is 10 ts'un.)

The original verbal solution: Multiply the diameter, the 2 ch'in 5 with itself. It has to be reduced by the product of the 7 by itself. Take the square root of the rest that is the width [1].

The reader can easily observe that the basis of the above calculation is equation (1).

Problem IX.14. Two persons are standing together. The proportion of $A$ 's walking is 7 , and the proportion of $B$ 's walking is 3 (i.e., the ratio of their walking speed is $7: 3)$. $B$ walks east, $A$ walks south $10 p u$, then walks diagonally (roughly) northeast, and meets $B$ How far do $A$ and $B$ walk?

Answer. $B$ walks $10 \frac{1}{2} p u, A$ walks diagonally $14 \frac{1}{2} p u$.

The original textual solution.

Calculate the following

$$
(7 \cdot 7+3 \cdot 3): 2, \quad 7 \cdot 7-(7 \cdot 7+3 \cdot 3): 2 \quad \text { and } 7 \cdot 3 .
$$

Then $A^{\prime}$ s diagonal move is

$$
\left(10 \cdot \frac{7 \cdot 7+3 \cdot 3}{2}\right):\left(7 \cdot 7-\frac{7 \cdot 7+3 \cdot 3}{2}\right)=14 \frac{1}{2},
$$

finally $B$ 's move to east is

$$
10 \cdot(7 \cdot 3):\left(7 \cdot 7-\frac{7 \cdot 7+3 \cdot 3}{2}\right)=10 \frac{1}{2} .
$$

What kind of considerations could be behind these calculations? What mathematical knowledge did the scribe know?

From Problems 1-3 we can conclude that they were familiar with the relation among the sides of any right-angled triangle, i.e., with the Theorem of Pythagoras as an empirical fact.

Let $x$ and $z$ denote $A$ 's walk to south and diagonally, moreover $y$ the way of $B$ to east. Then $x, y, z$ are sides of a right-angled triangle with perpendicular sides $x, y$. Thus we have either

$$
x^{2}+y^{2}=z^{2},
$$




$$
\text { "tmcs-klukovits" — 2013/10/21 — 15:33 — page } 262 \text { — } 8
$$

or

$$
\frac{y}{x+z}=\frac{3}{7} .
$$

Now we present two possible ways of the further calculations.

Method 1, using equations.

It can be performed without formulas (i.e., verbally), but for the readers' convinience we will use formulas/equations.

$A$ covers the distance

$$
z+x=z+10=\frac{7}{3} y
$$

Form the "theorem"

$$
x^{2}+y^{2}=10^{2}+y^{2}=z^{2} .
$$

If, in addition the scribe knew the identity $a^{2}-b^{2}=(a+b)(a-b)$ then $(7)$ can be written as

$$
(z+10)(z-10)=y^{2} .
$$

(6) and (7) imply

From the set of equations

$$
z-10=\frac{3}{7} y .
$$

$$
\begin{gathered}
z+10=\frac{7}{3} y, \\
z-10=\frac{3}{7} y, \\
y=2 z \frac{7 \cdot 3}{7^{2}+3^{2}} .
\end{gathered}
$$

Finally substituting this to (9), we have

$$
\begin{aligned}
z-10 & =2 \frac{3}{7} z \frac{7 \cdot 3}{7^{2}+3^{2}}=2 z \frac{3^{2}}{7^{2}+3^{2}}, \\
z \frac{7^{2}-3^{2}}{7^{2}+3^{2}} & =10, \\
z & =10\left(\frac{7^{2}+3^{2}}{7^{2}-3^{2}}\right) \\
& =10\left(\frac{7^{2}+3^{2}}{2}\right):\left(7^{2}-\frac{7^{2}+3^{2}}{2}\right), \\
z & =14 \frac{1}{2} .
\end{aligned}
$$




$$
\text { "tmcs-klukovits" — 2013/10/21 — 15:33 — page } 263 \text { — \#9 }
$$

To get $y$ put this to $(10)$ :

$$
\begin{aligned}
y & =2 \frac{10 \frac{7^{2}+3^{2}}{2}}{7^{2}-\frac{7^{2}+3^{2}}{2}} \cdot \frac{7 \cdot 3}{7^{2}+3^{2}} \\
& =10(7 \cdot 3):\left(7^{2}-\frac{7^{2}+3^{2}}{2}\right), \\
y & =10 \frac{1}{2}
\end{aligned}
$$

To calculate in this way, the scribe must be familiar not only with the "theorem' but also with the identity $u^{2}-v^{2}=(u+v)(u-v)$.

Method 2, using the result ascribed to Archytas.

Reformulate first the formulas: if $y=\alpha \beta(\alpha>\beta)$ is odd, $x=\frac{1}{2}\left(\alpha^{2}-\beta^{2}\right)$ and $z=\frac{1}{2}\left(\alpha^{2}+\beta^{2}\right)$, then $(x, y, z)$ is a Pythagorean triplet.

Using this we have

$$
x: y: z=\frac{\alpha^{2}-\beta^{2}}{2}: \alpha \beta: \frac{\alpha^{2}+\beta^{2}}{2} .
$$

Choose $\alpha=7, \beta=3$, and knowing that $x=10$ from (10) we have

$$
\frac{y}{x}=\frac{\alpha \beta}{\frac{\alpha^{2}-\beta^{2}}{2}} \Rightarrow y=\frac{10 \alpha \beta}{\frac{\alpha^{2}-\beta^{2}}{2}}=\frac{10 \alpha \beta}{\alpha^{2}-\frac{\alpha^{2}+\beta^{2}}{2}} .
$$

Substituting here $\alpha=7$ and $\beta=3$, here we get

$$
\begin{aligned}
y & =\frac{10(7 \cdot 3)}{\frac{7^{2}-3^{2}}{2}}=\frac{10(7 \cdot 3)}{7^{2}-\frac{7^{2}+3^{2}}{2}} \\
& =10 \cdot(7 \cdot 3):\left(7 \cdot 7-\frac{7 \cdot 7+3 \cdot 3}{2}\right)=10 \frac{1}{2} .
\end{aligned}
$$

Method 2 based on the book of Juskewich [5]. Both methods conclude to the use of numbers calculated by the Chinese scribe.

Behind this problem the triple $(20,21,29)$ appears. In solutions of other problems in Chapter IX the triples

$$
(5,12,13), \quad(8,15,17), \quad(7,24,25)
$$

appeared too. 


$$
\text { "tmcs-klukovits" — 2013/10/21 — 15:33 — page } 264 \text { — \#10 }
$$

\section{Digging deeper: Mesopotamia in the XVIIIth Century BC}

In the second quarter of the last century a considerable quantity of Mesopotamian clay tablets came into limelight containing mathematical content. They originate from two periods, from the time of the Hammurapi dynasty (Old Babylonian Empire) and from the Seleucid period. In our purposes the first collection has the main interest. This was the first part of the second millennium BC. Most of the clay tablets with mathematical content are now in the British Museum, in the Columbia University (the Plimpton collection) and in the Yale University.

In the later collection is the tablet YBC 7289. There is a square on it with the side marked by 30 and on the diagonal we can read $1 ; 24,51,10$ and 42; 25, 35 . These are sexagesimal numbers in the transcription due to O. Neugebauer [6]. The sequence $1 ; 24,51,10$ means $1+24 \cdot 60^{-1}+51 \cdot 60^{-2}+10 \cdot 60^{-3}$. This is a very good approximation of $\sqrt{2}$. Moreover, $42 ; 25,35=30 \cdot 1 ; 24,51,10$. This is a correct calculation of the diagonal based on the relation between the side and the diagonal of a square, i.e., what we call as Theorem of Pythagoras.

The next clay tablet, the Plimpton 322 , is more interesting.

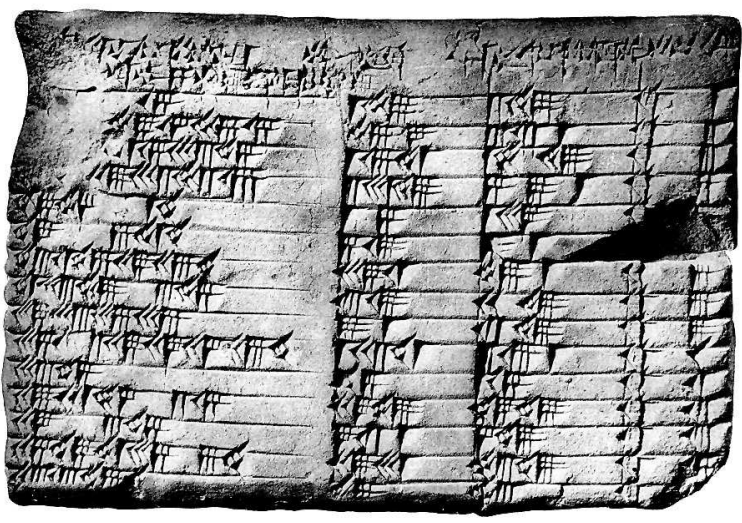

The tablet contains numerals in four columns written by cuneiform using only two signs, the wedge for 1 and for all integer power of $60 Y$, and the hinge for 10 and for $10 \cdot 60^{k}$ for any integer $k \ll$. 
Using the transcription above we have in [7]

$\begin{array}{cccc}\mathrm{I} & \mathrm{II}(=b) & \mathrm{III}(=d) & \mathrm{IV} \\ {[1,59,0,] 15} & 1,59 & 2,49 & 1 \\ {[1,56,56,] 58,14,50,6,15} & 56,7 & 3,12,1 & 2 \\ {[1,55,7,] 41,15,33,45} & 1,16,41 & 1,50,49 & 3 \\ {[1,] 5[3,1,] 0,29,32,52,16} & 3,31,49 & 5,9,1 & 4 \\ {[1,] 48,54,1,40} & 1,5 & 1,37 & 5 \\ {[1,] 47,6,41,40} & 5,19 & 8,1 & 6 \\ {[1,] 43,11,56,28,26,40} & 38,11 & 59,1 & 7 \\ {[1,] 41,33,59,3,45} & 13,19 & 20,49 & 8 \\ {[1,] 38,33,36,36} & 9,1 & 12,49 & 9 \\ 1,35,10,2,28,27,24,26,40 & 1,22,41 & 2,16,1 & 10 \\ 1,33,45 & 45 & 1,15 & 11 \\ 1,29,21,54,2,15 & 27,59 & 48,49 & 12 \\ {[1,] 27,0,3,45} & 7,12,1 & 4,49 & 13 \\ 1,25,48,51,35,6,40 & 29,31 & 53,49 & 14 \\ {[1,] 23,13,46,40} & 56 & 53 & 15\end{array}$

As can be seen, the left side of the tablet is broken and contains four columns. The right one (column IV) is simply the numbering of the rows. The headings of column II and III refere to breadth and diameter, respectively. We denoted them by $b$ and $d$. In the broken heading of column I there is a reference to diameter. The numbers in square brackets are substitutions, and the the signs of the missing sexagesimal digits (the Mesopotamian had no sign for the zero in that time) are also given [7].

On the basis of the Yale tablet mentioned above we can suppose, that the Mesopotamian scribe knew (verbally) equation (1), and using this he could calculate the numbers $h^{2}=d^{2}-b^{2}$ or $h=\sqrt{d^{2}-b^{2}}$. Column I contains the numbers $(b / h)^{2}$ or $1+(b / h)^{2}$. We cannot distinguish between these possibilities because almost all initial digits were broken.

All commentators agree that the three columns were probably preceded by at least three further columns containing the quotients $b / h=\beta$ and $d / h=\delta$, and the height $h$. Clearly, the the triples $(h, b, d)$ are Pythagorean.

We have to remark, that in the tablet there are some mistakes but there can easily be corrected and interpreted, see [7].

On the basis of Neugebauer and Sachs investigations van der Waerden set up a possibility how could the scribe calculate the numbers in the tablet $[9,10]$. The first observation is that the values $h$ have $2,3,5$ as prime divisors only. This 


$$
\text { "tmcs-klukovits" — 2013/10/21 — 15:33 — page } 266 \text { — \#12 }
$$

means that the values of $1 / h$ are finite sexagesimal fraction, i.e., the $h$-s are so called "regular numbers".

We can also suppose that the scribe started with $h=1$. After it he searched rational numbers $\beta$ and $\delta$ such that $\delta^{2}-\beta^{2}=1$, or in other words

$$
(\delta+\beta)(\delta-\beta)=1
$$

For suitable $p, q \in \mathbb{N}$ we have

$$
\delta+\beta=\alpha=\frac{p}{q}, \quad \delta-\beta=\alpha^{-1}=\frac{q}{p}
$$

and, by a methot frequently used in Mesopotamia,

$$
\delta=\frac{p^{2}+q^{2}}{2 p q}, \quad \beta=\frac{p^{2}-q^{2}}{2 p q},
$$

if we choose $h=2 p q, b=p^{2}-q^{2}$ and $d=p^{2}+q^{2}$. This chain can be found in several ancient books, e.g., in the Arithmetica of Diophantus.

Summarizing, the triple $(h, b, d)$ was derived a triple of the form of $(1, \beta, \delta)$. Line 11 confirms this argumentation. Namely, the three (remained) number in this line are 1,$0 ; 45,1 ; 15$. If we consider them as $\beta$ and $\delta$ and choose $2 p q=4$, then we get the well known triple $(4,3,5)$.

Neugebauer and Sachs $[6]$ pointed out that not only the numbers $h$ are "regular" but also $\alpha, \alpha^{-1}, p, q$ were "regular".

This arithmetic argumentation gives a partial answer to the selection of the triples on the tablet, i.e., crucial numbers are regular, the reciprocals if which are finite sexagesimal fractions. But it does not answer the order of the triples.

We can give a possible (geometrical) answer by examining the values $b / h$. In the first row $b=1,59$ while (the calculated) $h=2,0$. The quotient $1 ; 59 / 2,0$ is very close to 1 , i.e., the corresponding triangle is almost isosclenes, the half of a square, while the triangle corresponds to the last row has angles about $30^{\circ}$ and $60^{\circ}$, moreover the descent of the values $b / h$ is almost linear.

\section{Acknowledment}

The author is grateful to the referees for their helpful suggestions and comments. Moreover, a special thanks is given to Miklós Dormán for drawing a figure. 


$$
\text { "tmcs-klukovits" — 2013/10/21 — 15:33 — page } 267 \text { — \#13 }
$$

\section{References}

[1] Chiu Chang Suan Shu, Neun Bücher Arithmetischer Technik, Friedr. Vieweg and Sohn, Braunschweig, 1968.

[2] W. Geller, H. Küstner, M. Hellwich and H. Kästner, Mathematics at a Glance, VEB Bibligraphisches Institute, Leipcig, 1975.

[3] G. James and R. C. James (eds), Mathematics Dictionary, Van Nostrand, Princeton, Toronto, London and New York, 1959.

[4] T. L. Heath, Thirteen Books of Euclid's Elements, Dover (reprint), 1956.

[5] A. P. Juschkewitsch, Geschichte der Mathematik im Mittelalter, B. G. Teubner, Leipzig, 1964.

[6] O. Neugebauer and A. Sachs, Mathematical Cuneiform Texts, American Oriental Society, New Haven, 1945.

[7] O. Neugebauer, Exact Sciences in Antiquity, Brown University Press, Providence RI, 1970.

[8] A. Seidenberg, The Ritual Origin of Geometry, Archive for History of Exact Sciences 1 (1962), 488-527.

[9] B. L. van der Waerden, Science Awakening, Oxford University Press, New York, 1961.

[10] B. L. van der Waerden, Geometry and Algebra in Ancient Civilizations, Springer-Verlag, Berlin, Heidelberg, New York and Tokyo, 1983.

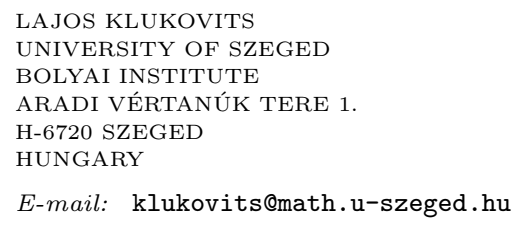

(Received July, 2013) 